\title{
Kininogen-1 as a protein biomarker for schizophrenia through mass spectrometry and genetic association analyses
}

\author{
Mingjia Yang ${ }^{1}$, Na Zhou ${ }^{2}$, Huiping Zhang ${ }^{3}$, Guojun Kang ${ }^{1}$, Bonan Cao ${ }^{1}$, Qi Kang ${ }^{1}$, Rixin Li $^{1}$, Xiaojing Zhu $^{1}$, \\ Wenwang Rao ${ }^{1,4}$, Qiong Yu ${ }^{\text {Corresp. } 1}$ \\ 1 Department of Epidemiology and Biostatistics, School of Public Health, Jilin University, Changchun, Jilin Province, China \\ 2 Department of Pharmacy, Hospital of Stomatology, Jilin University, Changchun, Jilin Province, China \\ 3 Departments of Psychiatry and Medicine (Biomedical Genetics), Boston University School of Medicine, Boston, USA \\ 4 Unit of Psychiatry, Faculty of Health Sciences, University of Macau, Macao, China \\ Corresponding Author: Qiong Yu \\ Email address: yuqiong@jlu.edu.cn
}

Background. Schizophrenia (SCZ) is a complex and severe mental illness. There is a lack of effective biomarkers for SCZ diagnosis. The aim of this study was to explore the possibility of using serum peptides for the diagnosis of SCZ as well as analyze the association of variants in genes coding for these peptides and SCZ.

Methods. After bead-based fractionation, the matrix-assisted laser desorption ionization/time-of-flight mass spectrometry (MALDI-TOF/MS) technique was used to identify peptides that showed different expressions between 166 SCZ patients and 201 healthy controls. Differentially expressed peptides were verified in a second set of sample (81SCZ patients and 103 healthy controls). The association of SCZ and three tagSNPs selected in genes coding for differentially expressed peptides was performed in 1,126 SCZ patients and 1,168 controls.

Results. The expression level of peptides with m/z 1945.07 was significant lower in SCZ patients than in healthy controls $(p<0.000001)$. The peptide with $\mathrm{m} / \mathrm{z} 1945.07$ was confirmed to be a fragment of Kininogen-1. In the verification tests, Kininogen-1 had a sensitivity of $95.1 \%$ and a specificity of $97.1 \%$ in SCZ prediction. Among the three tagSNPs (rs13037490, rs2983639, rs2983640) selected in the Cystatin 9 gene (CST9) which encodes peptides including Kininogen-1, tagSNP rs2983640 had its genotype distributions significantly different between SCZ patients and controls under different genetic models $(P<0.05)$. Haplotypes CG (rs2983639-rs2983640) and TCG (rs13037490-rs2983639-rs2983640) were significantly associated with SCZ (CG: $\mathrm{OR}=1.21,95 \% \mathrm{Cl}=1.02-1.44, P=0.032$; TCG: $\mathrm{OR}=24.85$, $95 \% \mathrm{Cl}=5.98-103.17, P<0.0001)$.

Conclusions. The present study demonstrated that SCZ patients had decreased expression of Kininogen-1 and genetic variants in Kininogen-1 coding gene CST9 were significantly associated with SCZ. The findings from both protein and genetic association studies suggest that Kininogen-1 could be a biomarker of SCZ. 


\section{Kininogen-1 as a protein biomarker for schizophrenia through mass}

\section{2 spectrometry and genetic association analyses}

3

4 Mingjia Yang ${ }^{1}$, Na Zhou ${ }^{2}$, Huiping Zhang ${ }^{3}$, Guojun Kang ${ }^{1}$, Bonan Cao ${ }^{1}$, Qi Kang ${ }^{1}$, Rixin Li $^{1}$, 5 Xiaojing Zhu ${ }^{1}$, Wenwang $\mathrm{Rao}^{1,4}$, Qiong $\mathrm{Yu}^{1}$

$7{ }^{1}$ Department of Epidemiology and Biostatistics, School of Public Health, Jilin University,

8 Changchun, Jilin Province, China

9 2Department of Pharmacy, Hospital of Stomatology, Jilin University, Changchun, Jilin Province, 10 China

$11{ }^{3}$ Departments of Psychiatry and Medicine (Biomedical Genetics), Boston University School of

12 Medicine, Boston, USA

$13{ }^{4}$ Unit of Psychiatry, Faculty of Health Sciences, University of Macau, Macao, China

Corresponding Author: Qiong $\mathrm{Yu}^{1}$

Email address: yuqiong@jlu.edu.cn 
21 Abstract

Background. Schizophrenia (SCZ) is a complex and severe mental illness. There is a lack of effective biomarkers for $\mathrm{SCZ}$ diagnosis. The aim of this study was to explore the possibility of using serum peptides for the diagnosis of $\mathrm{SCZ}$ as well as analyze the association of variants in genes coding for these peptides and SCZ.

Methods. After bead-based fractionation, the matrix-assisted laser desorption ionization/time-offlight mass spectrometry (MALDI-TOF/MS) technique was used to identify peptides that showed different expressions between $166 \mathrm{SCZ}$ patients and 201 healthy controls. Differentially expressed peptides were verified in a second set of sample (81SCZ patients and 103 healthy controls). The association of SCZ and three tagSNPs selected in genes coding for differentially expressed peptides was performed in 1,126 SCZ patients and 1,168 controls.

Results. The expression level of peptides with m/z 1945.07 was significant lower in SCZ patients than in healthy controls $(p<0.000001)$. The peptide with $\mathrm{m} / \mathrm{z} 1945.07$ was confirmed to be a fragment of Kininogen-1. In the verification tests, Kininogen-1 had a sensitivity of $95.1 \%$ and a specificity of $97.1 \%$ in SCZ prediction. Among the three tagSNPs (rs13037490, rs2983639, rs2983640) selected in the Cystatin 9 gene (CST9) which encodes peptides including Kininogen1, tagSNP rs2983640 had its genotype distributions significantly different between SCZ patients and controls under different genetic models $(P<0.05)$. Haplotypes CG (rs2983639-rs2983640) and TCG (rs13037490-rs2983639-rs2983640) were significantly associated with SCZ (CG: $\mathrm{OR}=1.21,95 \% \mathrm{CI}=1.02-1.44, P=0.032 ;$ TCG: $\mathrm{OR}=24.85,95 \% \mathrm{CI}=5.98-103.17, P<0.0001)$.

Conclusions. The present study demonstrated that SCZ patients had decrease expression of Kininogen-1 and genetic variants in Kininogen-1 coding gene CST9 were significantly associated with SCZ. The findings from both protein and genetic association studies suggest that Kininogen-1 could be a biomarker of SCZ.

45 6 47

\section{Introduction}


48

49

Schizophrenia (SCZ) is a complex psychotic disorder. It is among the top 25 mental illness in the world (Owen et al. 2016; Rodriguez 2015). Although the prevalence of SCZ is not high (0.5\%1.2\%) (Huang et al. 2019), the health and economic burden was enormous for patients and their families as well as the society (Chong et al. 2014). To date, the etiology and pathogenesis of SCZ are still unknown. The diagnosis of SCZ is mainly based on descriptive and observation criteria to assess SCZ symptoms described by patients and their family members or observed by medical workers. So far, no biochemical markers or screening tools are available for SCZ diagnosis (Santa et al. 2017). Thus, it is important to identify SCZ biomarkers with high specificity and sensitivity, especially those present in body fluids which are easily obtained.

It is well known that $\mathrm{SCZ}$ is a genetic disorder. Candidate gene or genome-wide association studies have been applied to identify genetic factors for SCZ. Even though genes carrying SCZassociated variants have been identified (Ghazaryan et al. 2016; Yoosefee et al. 2016), the mechanism by which how these genetic variants influence the vulnerability of individuals to $\mathrm{SCZ}$ is waiting to be explored. Since protein is the implementer of gene function, proteomic techniques such as mass spectrometry (MS) have been widely used in aiding in the diagnosis and identifying biomarkers of diseases including SCZ (Nascimento \& Martins-de-Souza 2015).

Mass spectrometry (MS) is an important technique for proteomics analysis. It separates and determines proteins according to the difference in mass-to-charge ratios $(\mathrm{M} / \mathrm{E})$ between different ions (Aebersold \& Goodlett 2001). A variety of MS techniques have been used in clinical application (Cho et al. 2015). For example, matrix-assisted laser desorption ionization/time-offlight mass spectrometry (MALDI-TOF/MS), a sensitive analytical technique, has been increasingly used to evaluate the expression of specific proteins and peptides to detect biomarkers of diseases including psychiatric disorders using biological samples from patients and controls (Ding et al. 2015; Huang et al. 2017; Kelley et al. 2018; Lo et al. 2016).

It is common that genes, proteins, and environment as well as the interaction among them lead to disease vulnerability. The exploration of disease biomarkers should conduct at multiple levels (Sokolowska et al. 2015). In the post-genomic era, genomics and proteomics studies are 
still two important areas that complement each other. Even though genetic variants may influence the structure and expression of proteins, variation in genes may not necessarily result in protein expression changes. However, changes in protein expression may reflect gene transcriptional. Recently, Comes et al. applied the proteomics approach in the identification of blood biomarkers for psychiatry disorders (Comes et al. 2018). They integrated proteomic findings from the peripheral blood and the genetic loci identified from enrichment analysis based on 30 published studies. With the availability of genomic data of a big sample the proteomic approach was demonstrated to be an essential method in discovery of biomarkers for disease including SCZ.

In present study, we initially used the MALDI-TOF/MS method to compare expression levels of serum proteins between SCZ patients and control subjects. We then screened candidate genes that code for differentially expressed proteins. Finally, we analyzed the association between polymorphisms of protein-coding gene and SCZ. Our aim was to explore etiology and diagnosis biomarkers of $\mathrm{SCZ}$ at both gene and protein levels.

\section{Materials and methods}

\section{Ethics statement}

Ethics approval for the study was granted by the Ethics Committee of the School of Public Health, Jilin University (2014-05-01). All participants provided written informed consent.

\section{Study overview}

To investigate the cause and find specific laboratory diagnostic biomarkers of SCZ, we used the bead-based fractionation method and the MALDI-TOF/MS technique to compare proteomes between first onset SCZ patients and healthy controls to identify specifically expressed proteins in SCZ patients. Verifications were conducted in another group of SCZ patients and healthy controls. Then, we searched for genes coding for differentially expressed proteins based on the National Center for Biotechnology Information (NCBI) database. Case-control genetic association analysis was used to explore the association between candidate gene polymorphisms and SCZ. 
102

103

104

105

106

107

108

109

110

111

\section{2}

113

114

115

116

117

118

119

120

121

122

123

124

125

126

127

128

\section{Subjects}

Serum samples for protein analysis were collected from 247 first-episode SCZ patients and 304 healthy volunteers. These first-episode SCZ patients have never taken psychiatric medications and did not receive electroconvulsive therapy in the past 3 months. DNA samples for casecontrol genetic association study were extracted from the peripheral blood of 1,126 SCZ patients and 1,168 healthy controls. All patients were recruited from the Changchun Mental Hospital as well as all healthy volunteers from the First Hospital of Jilin University.

All patients participated in present study were diagnosed by two independent psychiatrists. All healthy controls had no mental diseases or history of serious illness, and had not taken medications for at least two weeks. All subjects were Chinese Han in this study.

\section{Peptide extraction and MALDI-TOF/MS analysis}

Peptides were extracted by copper-chelated magnetic beads. Five microliter of homogeneous magnetic beads solution and $50 \mu 1$ of binding solution were added into a $0.2-\mathrm{ml}$ tube and mixed thoroughly. This tube was put in a magnetic bead separator (MBS) to fix the beads, and the supernatant was aspirated. Then $5 \mu 1$ of serum and $20 \mu 1$ of binding solution were added and mixed, and a MBS was used to fix the beads and the supernatant was aspirated again. After washing magnetic beads with $100 \mu \mathrm{l}$ of the washing solution, the beads were collected with MBS and the bound peptides were eluted using $20 \mu 1$ of the elution solution. For target preparation, one microliter of mixture containing one microliter of eluent and one microliter of the matrix (HCCA) solution was loaded onto a $600 \mu \mathrm{m}$-diameter spot size 384 MTP target plate (Bruker Daltonics) and left to dry.

Analysis of the processed samples was conducted by the Ultraflex ${ }^{\mathrm{TM}}$ III MALDI-TOF/MS instrument (Bruker Daltonics) equipped with an $\mathrm{N}_{2} 337 \mathrm{~nm}$ laser.

\section{Candidate gene and SNP selection}

The NCBI database was used to select the coding gene of Kininogen-1protein. Three tagSNPs (rs1303749, rs2983639, and rs2983640) in the candidate gene CST9 were selected using the Haploview program (http://hapmap.ncbi.nlm.nih.gov/). The Assay designer 3.1 was used to 
129

130

131

132

133

134

135

136

137

138

139

140

141

142

143

144

145

146

147

design the primers for genotyping these three tagSNPs. The primer sequences were as follows: rs 1303490: F: 5'-ACGTTGGATGAAGCAGTTCCCAGACTTAGG-3' R: 5'ACGTTGGATGTCTGGGAAAACTTCCCATTC-3'; rs2983639: F: 5'-ACG TTGGATGTCCATCTGCCCCTAAGTGAG-3' R: 5'-ACGTTGGATGACTACCT GACTATGGACTGC-3'; and rs2983640: F: 5'-ACGTTGGATGGTGTTCAAG GCAAACTCCAC-3' R: 5'-ACGTTGGATGTCTGAAGAGGAAATGGGTGG-3'.

\section{DNA extraction and SNP genotyping}

DNA was extracted from the peripheral blood using the ClotBlood DNA Kit (Cwbio, Beijing), and its concentration was detected by an ultraviolet spectrophotometer (Beckman, USA).

SNP genotyping was conducted using the Sequenom MassARRAY platform (San Diego, CA, USA). The whole process included PCR amplification, SAP reaction, PCR extension and desalination. The conditions of PCR was set as $94^{\circ} \mathrm{C}$ for 15 minutes to perform a hot-start, $94^{\circ} \mathrm{C}$ for 20 seconds to denature, $56^{\circ} \mathrm{C}$ for 30 seconds to anneal, $72^{\circ} \mathrm{C}$ for one minute for 45 cycles to extend, $72^{\circ} \mathrm{C}$ for three minutes to incubate. Then, the PCR product was incubated with SAP (Sequenom, Inc., San Diego, CA, USA) at $37^{\circ} \mathrm{C}$ for 40 minutes. After extension and desalination, the final products were analyzed by the MassARRAY software (Sequenom, USA).

\section{Statistical methods}

The protein spectrum analysis was conducted using the FlexAnalysis 3.0 software and the ClinProTools 2.1 software. The support vector machine (SVM) and k-nearest neighbor (K-NN) algorithms were used to perform class prediction, and after the calculation, statistical analysis results were cross-validated. For gene polymorphism analysis, demographic characteristics were compared using the $\chi^{2}$ analysis and rank sum tests. Hardy-Weinberg equilibrium (HWE) was examined by the Chi-square $\left(\chi^{2}\right)$ goodness-of-fit test. The allele frequency and genotype distribution of SNPs were analyzed by logistic regression analysis. Inheritance model and haplotype analyses were performed by the SNPStats program (https://www.snpstats.net/snpstatts), and the best model was selected based on the smallest akaike information criterion (AIC) (Akaike 1974). Data were analyzed using SPSS version 19.0. 
156 For all analyses, $\mathrm{p}<0.05$ (two-tailed) was considered statistically significant.

157

158

Results

159

160

161

162

163

164

165

166

167

168

169

170

171

172

173

174

175

176

177

178

179

180

181

182

\section{Comparison and identification of serum peptides}

\section{Peptide profiles of SCZ patients and healthy controls in training groups}

Serum peptide profiles of 166 first onset SCZ patients and 201 healthy controls were analyzed.

The mean age of $166 \mathrm{SCZ}$ patients (67 males and 99 females) and 201 control subjects (90 males and 111 females) was $33 \pm 13$ and $36 \pm 13$, respectively. Serum peptide patterns of SCZ patients were significantly different from those of healthy controls (supplementary Figure 1).

\section{Selection and identification of SCZ-specific serum peptides}

Through the t-test of ClinPro Tools software TM 2.1, with a p-value $<0.000001$, the $\mathrm{m} / \mathrm{z} 1945.07$ of peptides were significantly lower in patients compared to healthy controls (Figure 1(A)). Using the peptide with $\mathrm{m} / \mathrm{z} 1945.07$ to identify SCZ patients from healthy controls, the area under the curve (AUC) of the receiver operating characteristic (ROC) was 0.879 (Figure 1(B)).

The ion of m/z 1945.07 was confirmed to have the sequence NLGHGHKHERDQGHGHQ, which corresponded to Kininogen-1, with exactly the high molecular weight of kininogen. The sequence map was shown in Figure 2

\section{Peptide profiles of SCZ patients and healthy controls in testing groups}

Class prediction analyses were conducted in another group of SCZ patients and healthy controls.

There were 81 patients (35 males and 46 females) with mean age of $32 \pm 13$ and 103 healthy controls (45 males and 58 females) with mean age of $35 \pm 10$. The spectra analysis of the validation set classified 77 of the $81 \mathrm{SCZ}$ patients as SCZ positive and 100 of the 103 healthy controls as SCZ negative, revealing $95.1 \%$ sensitivity and $97.1 \%$ specificity.

Association between CST9 polymorphisms and SCZ

\section{Demographic characteristics and Hardy-Weinberg equilibrium}

Among 1,076 SCZ patients, there were 591 males and 476 females with mean age of $34 \pm 12$.

Among 1,151 healthy controls, there were 566 males and 585 females with mean age of $36 \pm 10$. 
183

184

185

186

187

188

189

190

191

192

193

194

195

196

197

198

199

200

201

202

203

204

205

206

207

208

209

SCZ patients and healthy controls were significantly different by age $(Z=-5.697, p<0.050)$ and $\operatorname{sex}\left(\chi^{2}=8.569, p<0.050\right)$. Except SNP rs2983640 in the patient group, genotype distributions of the three tagSNPs (rs13037490, rs2983639, and rs2983640) all met the norm of HWE in both patient and control groups $(\mathrm{p}>0.050)$.

\section{Allele frequency and genotype distribution analyses}

For SNP rs2983640, the difference in genotype distributions was significantly different between patients and controls $\left(\chi^{2}=9.53, p=0.009\right)$, but the allele frequency was not statistically different. For rs13037490 and rs2983639, no significant differences were found in both allele frequencies and genotype distributions between patients and controls $(p>0.05)$. Details were shown in Table 1.

\section{Inheritance modeling analysis}

For SNP rs2983640, under genetic model of codominant, recessive, or overdominant, the difference of genotype distributions between patient and control groups was significantly different (codominant: $\mathrm{OR}=1.35,95 \% \mathrm{CI}=1.03-1.78, P=0.0068$; recessive: $\mathrm{OR}=1.45$, $95 \% \mathrm{CI}=1.12-1.88, P=0.0051$; overdominant: $\mathrm{OR}=0.81,95 \% \mathrm{CI}=0.68-0.97, P=0.02)$. However, for SNPs rs 13037490 and rs2983639, under the four different genetic models, no significant difference was found between patients and controls $(p>0.050)$. The best genetic model for rs 13037490 and rs2983639 was recessive and overdominant, respectively. The best genetic model for rs13037490 and rs2983639 as well as the results from the difference models for rs2983640 were listed in Table 2.

\section{Haplotype analysis}

When haplotypes with frequency of more than 0.01 being considered, haplotypes CG (rs2983639-rs2983640) and TCG (rs13037490-rs2983639-rs2983640) were significantly associated with $\mathrm{SCZ}(\mathrm{CG}$ : $\mathrm{OR}=1.21,95 \% \mathrm{CI}=1.02-1.44, P=0.032$; TCG: $\mathrm{OR}=24.85$, 95\% $\mathrm{CI}=5.98-103.17, P<0.0001)$. No other haplotypes showed significant associations with SCZ.

\section{Discussion}

Even though there have been some studies reported that Kininogen-1 was significantly reduced in SCZ 
210

211

212

213

214

215

216

217

218

219

220

221

222

223

224

225

226

227

228

229

230

231

232

233

234

235

236

patients, our study was conducted in northern Han Chinese population, and we explored if variants in its coding gene were associated with SCZ. We confirmed that Kininogen-1 was a serum protein biomarker of SCZ, and it was lowly expressed in SCZ patients. SNP rs2983640 in CST9, one of the coding genes of Kininogen-1, was significantly associated with SCZ.

Additionally, the frequency of haplotypes carrying CST9 variants was significantly different between SCZ patients and healthy controls.

There are two types of Kininogens, low molecular weight kininogen (LMW) and high molecular weight kininogen (HMW) (Lalmanach et al. 2010), in mammals. In 1999, Irina firstly reported the association between $\mathrm{SCZ}$ and the kallikrein-kinin system (KKS). He found that plasma KKS system activity was increased in SCZ patients, and kallikrein in the brain of patients was significantly reduced. This finding suggested that KKS might play a role in blood-brain barrier and brain tissue damage, leading to the development of schizophrenia (Shcherbakova et al. 1999). Kininogen-1 is an important component of KKS. Together with factor XII, kallikreins, kinins, and kininases, it is involved in the regulation of multiple organs and diverse pathophysiological processes such as cardiovascular, renal, nervous system, tumor, thrombosis, atherosclerosis and cell proliferation, inflammation, and apoptosis (Albert-Weissenberger et al. 2013; Puchades et al. 2003; Regoli \& Gobeil 2015; Rhaleb et al. 2011).

HMW is a multifunctional glycoprotein in the plasma. It is synthesized by hepatocytes, and can be degraded to bradykinin (BK) under the action of kallikrein. BK receptors are also distributed in the central nervous system and play a role in diseases of the central nervous system (Chen et al. 2000). BK induces the synthesis and release of other inflammatory mediators in the central nervous system and the glial tissue, causing brain tissue damages or prolonged disturbance of blood-brain barrier function, which in turn can lead to brain damage (Ding-Zhou et al. 2002).

The production of kinins is influenced by the expression of kininogen. There was evidence about the mediatory role of kinins in the inflammatory response associated with different neurological disorders (Guevara-Lora 2012). In neurological diseases, such as Alzheimer's 
237

238

239

240

241

242

243

244

245

246

247

248

249

250

251

252

253

254

255

256

257

258

259

260

261

262

263

disease (AD), Parkinson's disease (PD), and multiple sclerosis (MS), proteins involved in kinin generation or kinin receptor function were overexpressed (Khandelwal et al. 2011; Lisak et al. 2011).

Kininogen is a member of the cystatin superfamily, which consists of type 1 cystatins (stefins), type 2 cystatins, and the kininogens. The results of this study indicated that compared with healthy controls, HMW was significantly reduced in schizophrenia patients. The deficiency of kininogen may lead the balance between proteases and protease inhibitors to be broken down in SCZ patients, resulting in the increase of homocysteine (Hcy) levels in the serum. Since Hcy is neurotoxic can affect the development of the central nervous system, it may be involved in the pathogenesis of SCZ. Several studies have shown that Hcy is significantly higher in SCZ patients than in healthy controls (Kale et al. 2010; Moustafa et al. 2014; Numata et al. 2015).

Kininogen is coded by the cystatin 9 gene (CST9), and cystatin 9 belongs to the Cystatins superfamily. It is one of 14 members of type 2 cystatins, which were found in human body compartments and fluids (Ochieng \& Chaudhuri 2010). In this study, we explored the association between three tagSNPs in CST9 and SCZ. Since age and gender of patients and controls were not well matched, we adjusted their confounding effect in the analysis. In the HWE test, SNP rs2983640 in the patient group did not meet the norm of HWE. This may be due to that rs2983640 is associated with SCZ. Findings from previous studies also indicate that SNPs that are not in HWE may contribute to complex diseases (Mayo 2008). For rs2983640, the genotype distribution was significantly different between patients and controls under the genetic model of codominant, recessive, or overdominant. Haplotypes CG (rs2983639-rs2983640) and TCG (rs13037490-rs2983639-rs2983640) were found to be associated with SCZ. All these results imply that polymorphisms of CST9 were associated with the risk of SCZ. Our genetic association studies not only indicated the association between CST9 variants and SCZ, but also confirmed that the kininogen-1 protein could be a biomarker for the diagnosis of SCZ.

However, there is still follow-up work waiting to be done in the future. For example, we need to conduct the study with a larger sample to ensure having sufficient statistical power. 
264 Subjects in this study were recruited in the northeast of China, and they were all Han Chinese.

265 We need to conduct studies in subjects from other areas or ethnicity groups. We also need to

266 study the effect of CST9 interaction with other genetic or environmental factors on the

267 occurrence of SCZ. Finally, candidate biomarkers should be validated in the absence of

268 interference from other diseases.

\section{Conclusions}

270 In summary, our study showed that using the MALDI-TOF/MS technique, Kininogen-1 could be 271 a biomarker to distinguish SCZ patients from healthy controls with a high sensitivity and 272 specificity. We also confirmed this at the gene level, i.e., variants in CST9, the coding gene of 273 Kininogen-1, were associated with the risk of SCZ. Both protein and genetic association studies 274 demonstrated that kininogen-1 could be a biomarker of SCZ. Definitely, our findings should be 275 validated in a larger sample and replicated in different populations.

\section{Acknowledgements}

278

279

280

281

282

283

284

285

286

287

288

289

290

291

292

293

294

We are grateful to Steven Limbara for checking the language of this manuscript.

\section{References}

Aebersold R, and Goodlett DR. 2001. Mass spectrometry in proteomics. Chemical Reviews 101:269-295.

Akaike H. 1974. A new look at the statistical model identification. Selected Papers of Hirotugu Akaike: Springer, 215-222.

Albert-Weissenberger C, Siren AL, and Kleinschnitz C. 2013. Ischemic stroke and traumatic brain injury: the role of the kallikrein-kinin system. Progress in Neurobiology 101-102:65-82.

Chen EY, Emerich DF, Bartus RT, and Kordower JH. 2000. B2 bradykinin receptor immunoreactivity in rat brain. Journal of Comparative Neurology 427:1-18.

Cho Y-T, Su H, Wu W-J, Wu D-C, Hou M-F, Kuo C-H, and Shiea J. 2015. Biomarker Characterization by MALDITOF/MS. Advances in Clinical Chemistry: Elsevier, 209-254.

Chong HY, Chaiyakunapruk N, Wu DBC, Lee KKC, and Chiou CF. 2014. PMH10-Global Economic Burden of Schizophrenia: A Systematic Review. Value in Health 17:357-373.

Comes AL, Papiol S, Mueller T, Geyer PE, Mann M, and Schulze TG. 2018. Proteomics for blood biomarker exploration of severe mental illness: pitfalls of the past and potential for the future. Transl Psychiatry 8:160. 
Ding-Zhou L, Marchand-Verrecchia C, Croci N, Plotkine M, and Margaill I. 2002. L-NAME reduces infarction, neurological deficit and blood-brain barrier disruption following cerebral ischemia in mice. European Journal of Pharmacology 457:137-146.

Ding Y-h, Guo J-h, Hu Q-y, Jiang W, and Wang K-z. 2015. Protein biomarkers in serum of patients with schizophrenia. Cell Biochemistry and Biophysics 72:799-805.

Ghazaryan H, Zakharyan R, Petrek M, and Arakelyan A. 2016. Annexin-A11 expression and its single nucleotide polymorphism (SNP) in schizophrenia. European Neuropsychopharmacology 26:S178-S179.

Guevara-Lora I. 2012. Kinin-mediated inflammation in neurodegenerative disorders. Neurochemistry International 61:72-78.

Huang TL, Lo LH, Shiea J, and Su H. 2017. Rapid and simple analysis of disease-associated biomarkers of Taiwanese patients with schizophrenia using matrix-assisted laser desorption ionization mass spectrometry. Clinica chimica acta; international journal of clinical chemistry 473:75.

Huang Y, Wang Y, Wang H, Liu Z, Yu X, Yan J, Yu Y, Kou C, Xu X, Lu J, Wang Z, He S, Xu Y, He Y, Li T, Guo W, Tian H, Xu G, Ma Y, Wang L, Wang L, Yan Y, Wang B, Xiao S, Zhou L, Li L, Tan L, Zhang T, Ma C, Li Q, Ding H, Geng H, Jia F, Shi J, Wang S, Zhang N, Du X, Du X, and Wu Y. 2019. Prevalence of mental disorders in China: a crosssectional epidemiological study. Lancet Psychiatry 6:211-224.

Kale A, Naphade N, Sapkale S, Kamaraju M, Pillai A, Joshi S, and Mahadik S. 2010. Reduced folic acid, vitamin B12 and docosahexaenoic acid and increased homocysteine and cortisol in never-medicated schizophrenia patients: implications for altered one-carbon metabolism. Psychiatry Research 175:47-53.

Kelley AR, Perry G, and Bach SB. 2018. Characterization of Proteins Present in Isolated Senile Plaques from Alzheimer's Diseased Brains by MALDI-TOF MS with MS/MS. ACS Chemical Neuroscience.

Khandelwal PJ, Herman AM, and Moussa CE. 2011. Inflammation in the early stages of neurodegenerative pathology. Journal of Neuroimmunology 238:1-11.

Lalmanach G, Naudin C, Lecaille F, and Fritz H. 2010. Kininogens: More than cysteine protease inhibitors and kinin precursors. Biochimie 92:1568-1579.

Lisak RP, Nedelkoska L, Studzinski D, Bealmear B, Xu W, and Benjamins JA. 2011. Cytokines regulate neuronal gene expression: differential effects of Th1, Th2 and monocyte/macrophage cytokines. Journal of Neuroimmunology 238:19-33.

Lo LH, Shiea J, and Huang TL. 2016. Rapid Detect ion of Alteration of Serum IgG in Patients with Schizophrenia after Risperidone Treatment by Matrix-Assisted Laser Desorption Ionization/ Time-of-Flight Mass Spectrometry. Rapid Communications in Mass Spectrometry Rcm 30:2645-2649.

Mayo O. 2008. A century of Hardy-Weinberg equilibrium. Twin Research \& Human Genetics 11:249-256.

Moustafa AA, Hewedi DH, Eissa AM, Frydecka D, and Misiak B. 2014. Homocysteine levels in schizophrenia and affective disorders-focus on cognition. Frontiers in Behavioral Neuroscience 8:343.

Nascimento JM, and Martins-de-Souza D. 2015. The proteome of schizophrenia. npj Schizophrenia 1:14003.

Numata S, Kinoshita M, Tajima A, Nishi A, Imoto I, and Ohmori T. 2015. Evaluation of an association between plasma total homocysteine and schizophrenia by a Mendelian randomization analysis. BMC Medical Genetics 16:54.

Ochieng J, and Chaudhuri G. 2010. Cystatin superfamily. Journal of Health Care for the Poor and Underserved 21:51-70.

Owen MJ, Sawa A, and Mortensen PB. 2016. Schizophrenia. Lancet 388:86-97.

Peer) reviewing PDF | (2019:03:35682:2:2:NEW 18 Jun 2019) 
336

337

338

339

340

341

342

343

344

345

346

347

348

349

350

351

352

353

354

355

356

357
Puchades M, Hansson SF, Nilsson CL, Andreasen N, Blennow K, and Davidsson P. 2003. Proteomic studies of potential cerebrospinal fluid protein markers for Alzheimer's disease. Brain Research: Molecular Brain Research 118:140-146.

Regoli D, and Gobeil F, Jr. 2015. Critical insights into the beneficial and protective actions of the kallikrein-kinin system. Vascular Pharmacology 64:1-10.

Rhaleb NE, Yang XP, and Carretero OA. 2011. The kallikrein-kinin system as a regulator of cardiovascular and renal function. Compr Physiol 1:971-993.

Rodriguez A. 2015. Global, regional, and national incidence, prevalence, and years lived with disability for 301 acute and chronic diseases and injuries in 188 countries, 1990-2013: a systematic analysis for the Global Burden of Disease Study 2013. Lancet 386:743-800.

Santa C, Coelho JF, Madeira N, and Manadas B. 2017. Circulating biomarkers in schizophrenia: a proteomics perspective.

Shcherbakova I, Neshkova E, Dotsenko V, Platonova T, Shcherbakova E, and Yarovaya G. 1999. The possible role of plasma kallikrein-kinin system and leukocyte elastase in pathogenesis of schizophrenia. Immunopharmacology 43:273-279.

Sokolowska I, Wetie AGN, Wormwood K, Thome J, Darie CC, and Woods AG. 2015. The potential of biomarkers in psychiatry: focus on proteomics. Journal of Neural Transmission 122:9-18.

Yoosefee S, Ananloo ES, Joghataei M-T, Karimipour M, Hadjighassem M, Mohaghghegh H, Tehrani-Doost M, Rahimi A-A, Abdolmaleky HM, and Hatami M. 2016. Association Between Neuregulin-1 Gene Variant(rs2439272) and Schizophrenia and Its Negative Symptoms in an Iranian Population. Iranian journal of psychiatry 11:147. 
Figure 1

\section{Comparisonof $\mathrm{m} / \mathrm{z} 1945.07$ expression level between schizophrenia patients and healthycontrols}

A: Distribution of serum peptide with $\mathrm{m} / \mathrm{z} 1945.07$ between schizophrenia (red) and healthy controls (green); B :Receiver operating characteristic (ROC) of m/z 1945.07 to distinguish schizophrenia patients from healthy controls;
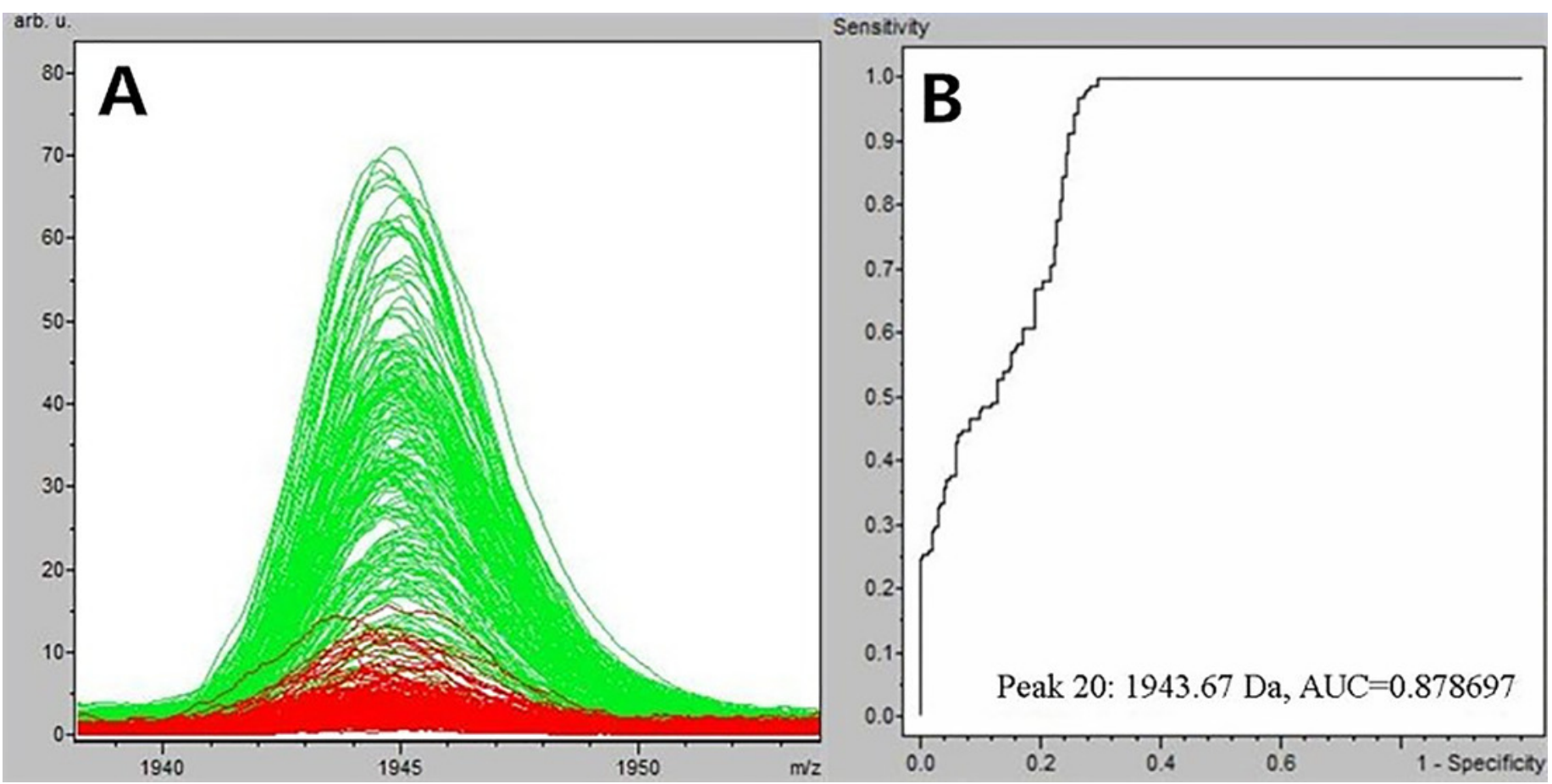
Figure 2

The sequence map of $\mathrm{m} / \mathrm{z} 1945.07$

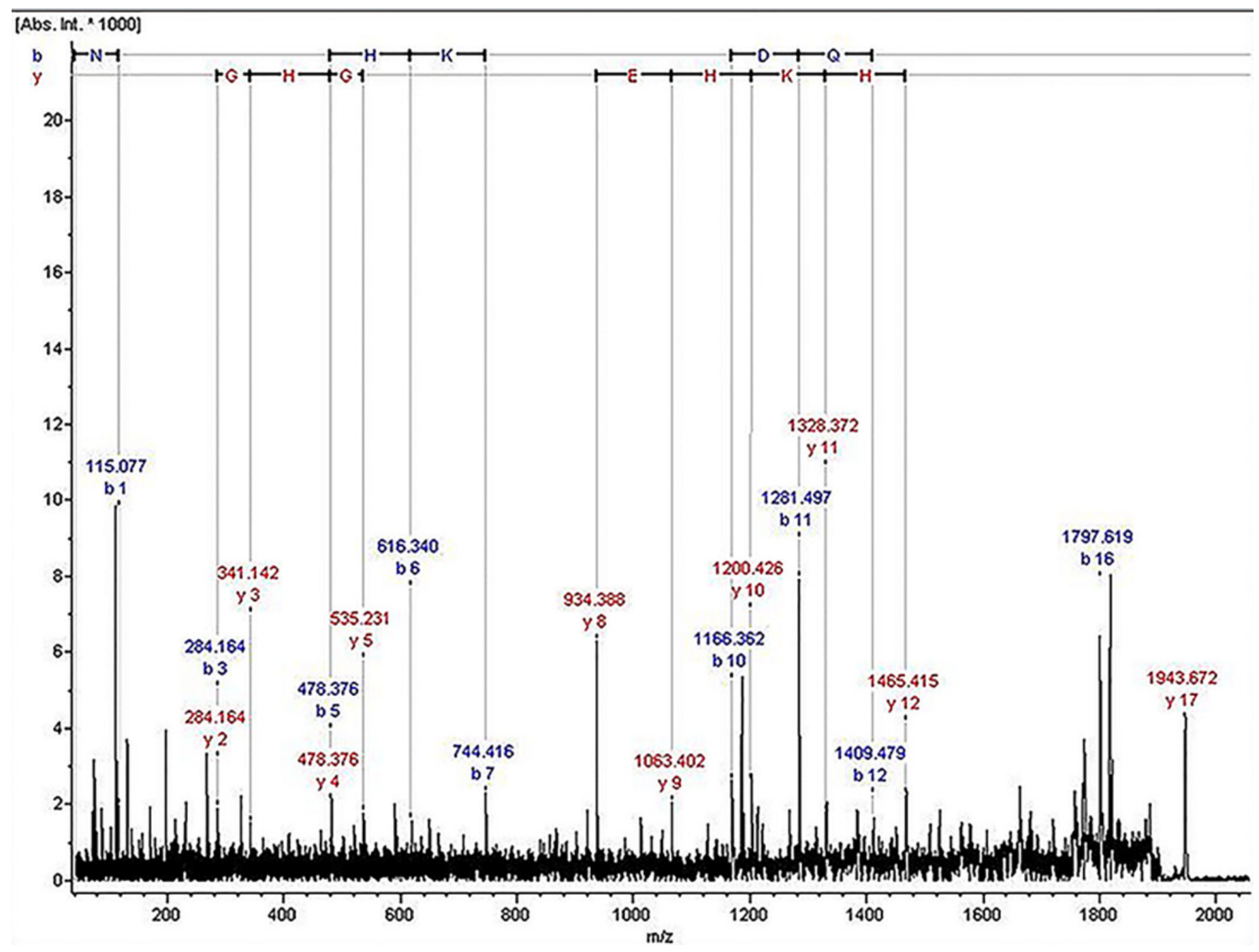




\section{Table $\mathbf{1}$ (on next page)}

Comparison of allele frequencies and genotypedistributions of three tagSNPs between schizophrenia patients and healthy controls

Note : $P$ values were adjusted for age and gender. 
1

2 Table 1 Comparison of allele frequencies and genotype distributions of three tagSNPs between 3 schizophrenia patients and healthy controls

\begin{tabular}{cllllll}
4 & SNP & Genotype/Allele & SCZ(\%) & Control(\%) & $\chi^{2}$ & $P$ \\
\cline { 2 - 6 } 6 & rs13037490 & T/T & $807(75)$ & $864(76)$ & 0.71 & 0.702 \\
7 & T/C & $245(23)$ & $264(23)$ & & \\
8 & C/C & $19(2)$ & $16(2)$ & & \\
9 & T & $1859(87)$ & $1992(87)$ & 0.13 & 0.719 \\
10 & rs2983639 & C/C & $283(13)$ & $296(13)$ & & \\
11 & C/T & $682(65)$ & $713(62)$ & 4.19 & 0.123 \\
12 & T/T & $320(30)$ & $393(34)$ & & \\
13 & C & $49(5)$ & $44(4)$ & & \\
14 & T & $1684(80)$ & $1819(79)$ & 0.67 & 0.412 \\
& A/A & $418(20)$ & $481(21)$ & & \\
& rs2983640 & $424(44)$ & $492(43)$ & 9.95 & 0.007 \\
& A/G & $398(41)$ & $528(46)$ & & \\
& G/G & $143(15)$ & $124(11)$ & & \\
& A & $1246(65)$ & $1512(66)$ & 1.13 & 0.287 \\
& G & $684(35)$ & $776(34)$ & & \\
\hline
\end{tabular}




\section{Table 2 (on next page)}

\section{Comparison ofinherence models between patients and controls}

Note : OR $(95 \% \mathrm{Cl})$ and $P$ values were adjusted for age and gender. 


\begin{tabular}{|c|c|c|c|c|c|c|c|}
\hline SNP & Model & Genotype & SCZ(\%) & Control(\%) & OR(95\%Cl) & $P$ & AIC \\
\hline \multirow[t]{3}{*}{ rs13037490 } & Recessive & T/T-T/C & 1024(98.2) & $1128(98.6)$ & 1.00 & 0.4 & 3026.5 \\
\hline & & $\mathrm{C} / \mathrm{C}$ & $19(1.8)$ & $16(1.4)$ & $1.34(0.68-$ & & \\
\hline & & & & & 2.62) & & \\
\hline \multirow[t]{3}{*}{ rs2983639 } & Overdominant & C/C-T/T & $724(69.5)$ & $757(65.8)$ & 1.00 & 0.058 & 3005.8 \\
\hline & & $\mathrm{C} / \mathrm{T}$ & $317(30.4)$ & $393(34.2)$ & $0.84(0.70-$ & & \\
\hline & & & & & 1.01) & & \\
\hline \multirow[t]{11}{*}{ rs2983640 } & Codominant & $A / A$ & $419(43.8)$ & $492(43)$ & 1.00 & 0.0068 & 2864.5 \\
\hline & & $\mathrm{G} / \mathrm{A}$ & $394(41.2)$ & $528(46.1)$ & $0.87(0.72-$ & & \\
\hline & & & & & $1.05)$ & & \\
\hline & & $\mathrm{G} / \mathrm{G}$ & $143(15)$ & $124(10.8)$ & $1.35(1.03-$ & & \\
\hline & & & & & $1.78)$ & & \\
\hline & Recessive & A/A-G/A & $813(85)$ & $1020(89.2)$ & 1.00 & 0.0051 & 2864.7 \\
\hline & & $\mathrm{G} / \mathrm{G}$ & $143(15)$ & $124(10.8)$ & $1.45(1.12-$ & & \\
\hline & & & & & $1.88)$ & & \\
\hline & Overdominant & $A / A-G / G$ & $562(58.8)$ & $616(53.9)$ & 1.00 & 0.02 & 2867.1 \\
\hline & & $\mathrm{G} / \mathrm{A}$ & $394(41.2)$ & $528(46.1)$ & $0.81(0.68-$ & & \\
\hline & & & & & $0.97)$ & & \\
\hline
\end{tabular}

2 Note : OR $(95 \% \mathrm{Cl})$ and $P$ values were adjusted for age and gender.

3

4

5 